\title{
REGULATORY EFFECTS OF INTERLEUKIN-10 ON LUNG ISCHEMIA-REPERFUSION INJURY
}

Michael J. Eppinger, MD ${ }^{a, b}$

Peter A. Ward, MD ${ }^{c}$

Steven F. Bolling, $\mathrm{MD}^{\mathrm{a}}$

G. Michael Deeb, $\mathrm{MD}^{\mathrm{a}}$
Objective: Interleukin-10, a cytokine with antiinflammatory activities, was studied to determine its effects on development of early lung reperfusion injury. Methods: Adult male rats underwent 90 minutes of left lung ischemia followed by 4 hours of reperfusion. Time-matched sham-operated control rats underwent hilar dissection but not lung ischemia. Lung injury was measured by vascular permeability to bovine serum albumin tagged with iodine 125. To evaluate the effect of exogenous interleukin-10, additional animals received interleukin-10 intravenously before ischemia. To assess the role of endogenous interleukin-10, animals received rabbit antimouse interleukin-10 immunoglobin $G$ (or preimmune rabbit immunoglobin $G$ ) before ischemia. Results: Compared with sham control rats, ischemiareperfusion control rats demonstrated significantly more lung injury. Animals receiving interleukin-10 had significantly less lung injury than did ischemia-reperfusion control rats. Animals receiving antiinterleukin-10 had significantly more lung injury than did animals receiving preimmune immunoglobin G. Alveolar macrophages from animals after 90 minutes of lung ischemia produced more tumor necrosis factor- $\alpha$ in culture than did unstimulated macrophages; this production was reduced significantly by the addition of interleukin-10 to the culture medium. Conclusion: Endogenous interleukin-10 has a protective effect against early lung reperfusion injury, and interleukin-10 administration can reduce lung reperfusion injury, perhaps in part through its ability to reduce production by alveolar macrophages of tumor necrosis factor- $\alpha$, a known proinflammatory cytokine. (J Thorac Cardiovasc Surg 1996;112:1301-6)
From the Section of Thoracic Surgery ${ }^{\mathrm{a}}$ and Department of Pathology, ${ }^{c}$ University of Michigan Medical Center, Ann Arbor, Mich., and Wright State University Department of Surgery, ${ }^{\mathrm{b}}$ Dayton, Ohio.

This work was supported in part by National Institutes of Health grant GM 29507 (P. A. W.). M. J. E. is supported by the United States Air Force.

Opinions and conclusions in this paper are those of the authors and are not intended to represent the official position of the Department of Defense, the United States Air Force, or any other government agency.

Read at the Seventy-sixth Annual Meeting of The American Association for Thoracic Surgery, San Diego, Calif., April 28-May 1, 1996.

Received for publication May 6, 1996; revisions requested June 26, 1996; revisions received July 12, 1996; accepted for publication July 15, 1996.

Address for reprints: G. Michael Deeb, MD, University of Michigan Hospitals, 1500 E. Medical Center Dr., 2124F Taubman Center TC/0344, Ann Arbor, MI, 48109.12/6/76516

Copyright (C) 1996 by Mosby-Year Book, Inc.

$12 / 6 / 76516$ ung transplantation has emerged as a viable -therapy for patients with end-stage lung disease. Difficulties in maintaining optimal graft function continue, however, to compromise patient outcomes. Early episodes of rejection and infection impair the function of lung allografts and diminish the long-term prognosis for patients undergoing lung transplantation. During the intraoperative or early postoperative period, lung dysfunction from technical difficulties or lung reperfusion injury threatens graft viability and patient survival. ${ }^{1}$ There is evidence that significant reperfusion injury predicts a worse outcome for long-term graft survival. ${ }^{2}$

Interleukin-10 (IL-10) has been identified as a potent inhibitor of early-phase inflammatory cytokine production. ${ }^{3,4}$ As such, it may serve to dampen the acute upregulation of inflammatory cytokines that occurs in response to many different stimuli. In the setting of orthotopic liver transplantation, IL-10 release has been found to be upregulated dramatically during the early reperfusion period, ${ }^{5}$ leading to speculation about its eventual effects both on reper- 
fusion injury and on the development of early graft rejection.

With a rat lung model of ischemia-reperfusion, we previously demonstrated that significant injury occurs in the reperfused lung in a bimodal pattern during the first 4 hours of reperfusion. ${ }^{6}$ With this model, we sought to determine the effects of IL-10 on lung reperfusion injury both through the administration of recombinant IL-10 and through antibody blockade of endogenous IL-10. We also previously found a significant role for tumor necrosis factor- $\alpha$ (TNF- $\alpha$ ) in the development of lung reperfusion injury. ${ }^{7}$ Consequently, we attempted to determine whether any protective effects of IL-10 on lung reperfusion injury might be linked in part to downregulation of TNF- $\alpha$ production in vivo.

\section{Materials and methods}

Reagents. All reagents were purchased from Sigma Chemical Company (St. Louis, Mo.) unless otherwise specified. Recombinant murine IL-10 was a generous gift from Dr. Maureen Howard (DNAX Research Institute, Palo Alto, Calif.).

Animal model. Pathogen-free adult male Long-Evans rats (300 to $350 \mathrm{~g}$; Harlan Sprague Dawley, Inc. Indianapolis, Ind.) were used for all experiments. Experimental protocols were approved by the University of Michigan Committee on the Use and Care of Animals. Animals were initially anesthetized intraperitoneally with 25 to 40 $\mathrm{mg}$ pentobarbital. The animals were then shaved, orotracheally intubated with a 14-gauge catheter, and placed on a Harvard rodent ventilator (Harvard Apparatus, Inc., South Natick, Mass.) with supplemental oxygen (60\%) at a rate of 75 cycles/min, with a maximum $10 \mathrm{~cm} \mathrm{H}_{2} \mathrm{O}$ peak pressure and a maximum $2 \mathrm{~cm} \mathrm{H}_{2} \mathrm{O}$ positive end-expiratory pressure. All animals received $0.4 \mathrm{mg}$ intramuscular atropine after being anesthetized. Animals received halothane through the ventilator system $(0.4 \%$ to $1 \%)$ to maintain anesthesia as necessary during the course of the experiment. Each animal was then placed on its right side and a left anterolateral thoracotomy was carried out through the fifth interspace. The left pulmonary hilum was stripped of all neural, vascular, lymphatic, and connective tissue, skeletonizing the left bronchus, pulmonary artery, and pulmonary vein. The inferior pulmonary ligament was divided as it entered the hilum. All dissection was carried out under an operating microscope. Each animal received $50 \mathrm{U}$ heparin in saline solution intravenously (total volume $500 \mu$ i) through the penile vein. Five minutes after infusion of the heparin, the left pulmonary artery, bronchus, and pulmonary vein were sequentially occluded with noncrushing microvascular clamps. The lungs were kept moist with intermittent application of warm normal saline solution, and the wounds were covered with plastic film to prevent excessive fluid loss. Periods of ischemia were held constant at 90 minutes. At the end of the period of ischemia, the clamps were removed from the vein, bronchus, and artery, in that order, and the lungs were allowed to ventilate and reperfuse for 4 hours. Animals received $0.5 \mathrm{ml}$ normal saline solution by subcutaneous injection for each hour of reperfusion to maintain hydration. Animals were killed at the end of reperfusion by clamping of the right and left superior vena cavae and of the inferior vena cava. The left atrial appendage was then amputated, and the lungs were flushed with $30 \mathrm{ml}$ saline solution by gravity infusion at $30 \mathrm{~cm} \mathrm{H}_{2} \mathrm{O}$, through the right ventricle. Blood samples were obtained from the inferior vena cava just before the animals were killed. The left lung was then removed for analysis as outlined below. Animals undergoing ischemia and reperfusion are designated as the positive control group $(n=6)$. Time-matched shamoperated control rats (sham control group, $n=4$ ) underwent the identical procedure, except that the microvascular clamps were not applied to the hilar structures.

Lung permeability assessment. To quantify injury to the lung as a result of ischemia and reperfusion, lung vascular permeability was measured in the following manner. Bovine serum albumin (BSA) labeled with iodine 125, prepared by a standard chloramine-T method, was diluted to approximately 3 to $5 \mu \mathrm{Ci} / \mathrm{ml}$ in $1 \% \mathrm{BSA} /$ phosphatebuffered saline solution (PBS). An 800,000 counts/min quantity of ${ }^{125}$ I-BSA was then brought to a final volume of $500 \mu \mathrm{l}$ in PBS for injection. At the time of unclamping the hilar structures or at the comparable time in the sham control group, the mix was injected into the animals through the penile vein. Immediately before each animal was killed, $1 \mathrm{ml}$ blood was drawn from the inferior vena cava for counting. After the animal was killed and its pulmonary vasculature was flushed, the left lung was excised for counting. Each lung specimen was weighed, and the lungs and blood were counted separately in a gamma counter. All results are expressed the ratio of counts per minute $1 \mathrm{gm}$ lung tissue to $1 \mathrm{ml}$ blood. This ratio provided a reliable measure of microvascular permeability.

IL-10 studies. To determine the effect of exogenously administered IL-10, additional animals $(n=4)$ received $10 \mu \mathrm{g}$ recombinant murine IL-10 in PBS along with the heparin 5 minutes before induction of ischemia. These animals then underwent the same ischemia-reperfusion protocol, with microvascular permeability determined as previously described.

Protein $G$ purified immunoglobin $G$ ( $(\mathrm{gGG})$ fractions of whole serum either from nonimmunized (preimmune) rabbits or from rabbits immunized against recombinant murine IL-10 were used to examine the effects of endogenously produced IL-10 on lung ischemia-reperfusion injury. Animals undergoing the same ischemia-reperfusion protocol received $200 \mu \mathrm{g}$ of either rabbit antimouse IL-10 IgG or preimmune IgG $(n=5$ each) before the initiation of ischemia. Microvascular permeability was determined as previously described.

Alveolar macrophage production of TNF- $\alpha$. Alveolar macrophages were obtained by bronchoalveolar lavage with Dulbecco's PBS from the lungs of either normal, unstimulated rats $(n=4)$ or selectively from the left lungs of animals subjected to 90 minutes of left lung ischemia $(n=8)$. After lavage, the cell suspensions were spun down and resuspended in RPMI medium containing $10 \%$ fetal bovine serum with or without $250 \mathrm{ng} / \mathrm{ml}$ recombinant murine IL-10. An aliquot was then obtained for cell 


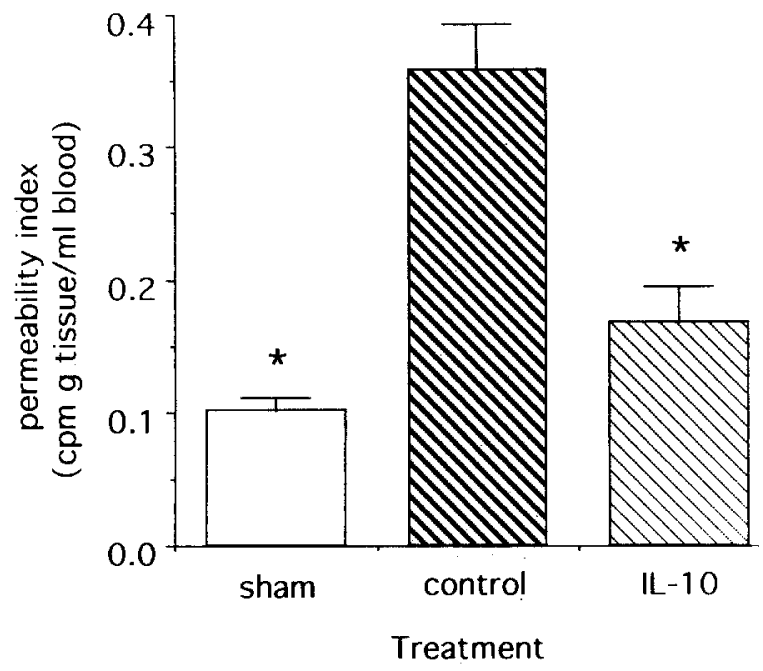

Fig. 1. Ischemic lung permeability index. Sham, Shamoperated control group; control, positive control group undergoing 90 minutes of left lung ischemia and 4 hours of reperfusion; $I L-10$, experimental group undergoing ischemia and 4 hours of reperfusion after infusion of 10 $\mu \mathrm{g}$ recombinant mouse IL-10. Asterisk indicates $p<0.01$ vs control group.

counting with a hemocytometer. A sample of $1 \times 10^{5}$ macrophages in a volume of $500 \mu \mathrm{l}$ was then pipetted into a culture dish and incubated for 4 hours at $37^{\circ} \mathrm{C}$ in $5 \%$ carbon dioxide in oxygen. At the end of the incubation period, the medium was removed and spun down to remove any cells; the supernatant was then pipetted off and stored at $-80^{\circ} \mathrm{C}$. The supernatant fluid was later analyzed for TNF- $\alpha$ content by means of a cytotoxicity bioassay (WEHI 164, subclone 13).

Northern blot analysis. Total ribonucleic acid (RNA) was extracted from lung homogenates with a phenol/ guanidine isothiocyanate/chloroform-based technique (TRIzol Reagent; Life Technologies, Inc., Gaithersburg, Md.) followed by isopropanol precipitation. A sample with $12 \mu \mathrm{g}$ total RNA per lane was fractionated on a $1 \%$ agarose formaldehyde gel and blotted to a nylon membrane (Zetabind; CUNO, Inc., Meriden, Conn.) Consistency of gel loading was assessed by staining of $18 \mathrm{~S}$ and $28 \mathrm{~S}$ ribosomal RNA bands on the nylon membrane with methylene blue. Radiolabeled probes (deoxycytidine triphosphate labeled with phosphorus 32) were generated by using polymerase chain reaction primers specific for the full-length open-reading frame complementary deoxyribonucleic acid template of rat TNF- $\alpha$. Radioactivity of the probes was determined by scintillation counting, and $1.5 \times 10^{7}$ counts/min radiolabeled probe was applied to the blot, followed by hybridization at $65^{\circ} \mathrm{C}$ overnight. Autoradiography of the blots was carried out at $-70^{\circ} \mathrm{C}$ on Kodak X-OMAT-AR film (Eastman Kodak Company, Rochester, N.Y.). Densitometry of autoradiographs was performed by means of a Fotodyne densitometer (Foto-

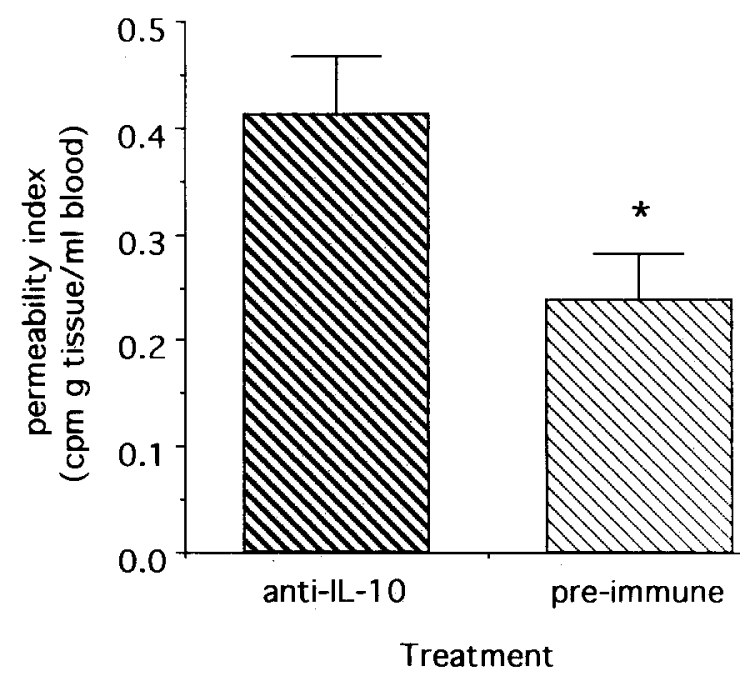

Fig. 2. Ischemic lung permeability index in animals undergoing ischemia and reperfusion after receiving either rabbit antimouse IL-10 $\operatorname{lgG}$ or preimmune rabbit IgG. Asterisk indicates $p=0.04$ vs anti-IL-10.

dyne, Inc., Hartland, Wis.) with AMBIS software (Ambis, Inc., San Diego, Calif.).

Statistical analysis. All data are presented as mean ( \pm standard error of the mean) unless otherwise designated. Comparisons between groups were made with a one-way analysis of variance, and Tukey's procedure was used to determine significant differences between groups. Single two-sample comparisons were made with a two-tailed, unpaired Student's $t$ test. Statistical significance was set at a $p$ level less than 0.05 .

\section{Results}

Ischemia-reperfusion lung injury. For lung injury studies, animals underwent the standard 90 minutes of ischemia followed by 4 hours of reperfusion (positive control group, $n=6$ ). Time-matched sham control group animals underwent similar operative procedures in the absence of ischemia and were then left attached to the ventilator for a duration corresponding to the 90 -minute ischemic time plus 4 hours $(n=4)$. Microvascular permeability was measured as described previously, by means of sequestration of ${ }^{125}$ I-BSA. Compared with the sham group, the positive control group demonstrated significantly more lung injury at the end of the 4-hour reperfusion time course (mean permeability index $0.358 \pm 0.035$ vs $0.102 \pm 0.009, p<$ 0.01 ). Animals receiving recombinant IL-10 before the start of ischemia $(n=4)$ demonstrated significantly less lung injury than did the positive control group (mean permeability index $0.167 \pm 0.028, p<$ 


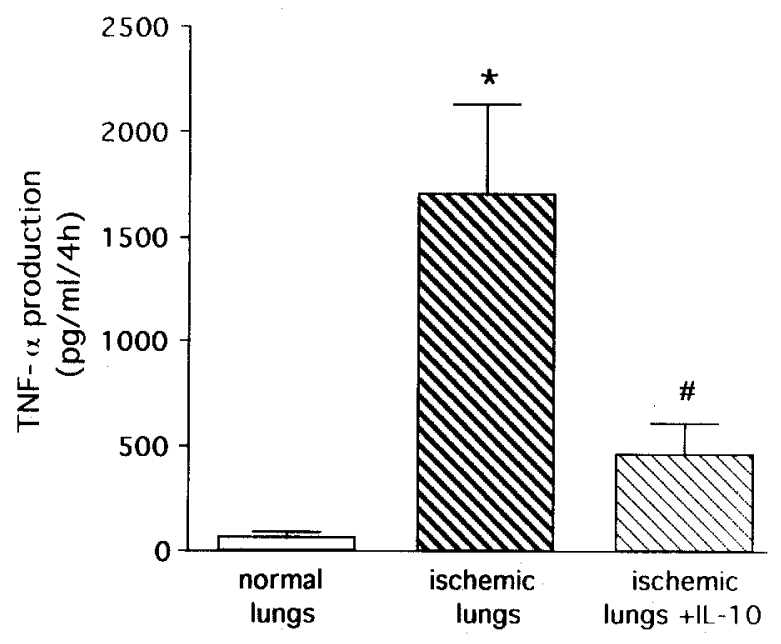

alveolar macrophage groups

Fig. 3. TNF- $\alpha$ concentration in supernatant of cultured alveolar macrophages after 4 hours at $37^{\circ} \mathrm{C}$, measured by WEHI cell cytotoxicity bioassay. Unstimulated alveolar macrophages were lavaged from normal animals or selectively lavaged from left lungs after 90 minutes of in vivo ischemia. Macrophages were then cultured for 4 hours in medium alone or in medium containing $250 \mathrm{ng} / \mathrm{ml} \mathrm{recom-}$ binant mouse IL-10. Asterisk indicates $p<0.01$ vs normal lung macrophages; pound sign indicates $p<0.05$ vs ischemic lung macrophages in medium without IL-10.

0.01 , Fig. 1). These data demonstrate the ability of exogenous IL-10 to lessen the severity of lung ischemia-reperfusion injury during this time course.

Among animals treated with intravenous $\operatorname{IgG}$ before the start of ischemia, there was significantly more lung injury in animals receiving antibody against recombinant IL-10 than in animals receiving preimmune antibody (mean permeability index $0.411 \pm 0.056$ vs $0.237 \pm 0.044, p=0.04$, Fig. 2). Blocking the effects of endogenous IL-10 produced in response to lung ischemia-reperfusion exacerbates the associated injury.

Alveolar macrophage TNF- $\alpha$ production. Alveolar macrophages from lungs subjected to $90 \mathrm{~min}$ utes of ischemia produced significantly more TNF- $\alpha$ in 4 hours than did macrophages lavaged from the lungs of normal animals $(1695 \pm 432$ vs $57 \pm 37 \mathrm{pg}$ $\left.\mathrm{ml}^{-1} \cdot 4 \mathrm{hr}^{-1}, p<0.01\right)$. When alveolar macrophages from ischemic lungs were cultured in the presence of recombinant IL-10, they produced significantly less TNF- $\alpha$ than did macrophages from ischemic lungs cultured in medium alone (458 \pm 158 vs $1695 \pm 432 \mathrm{pg} \cdot \mathrm{ml}^{-1} \cdot 4 \mathrm{hr}^{-1}, p<0.05$, Fig. 3). These data confirm the high level of TNF- $\alpha$ produc-

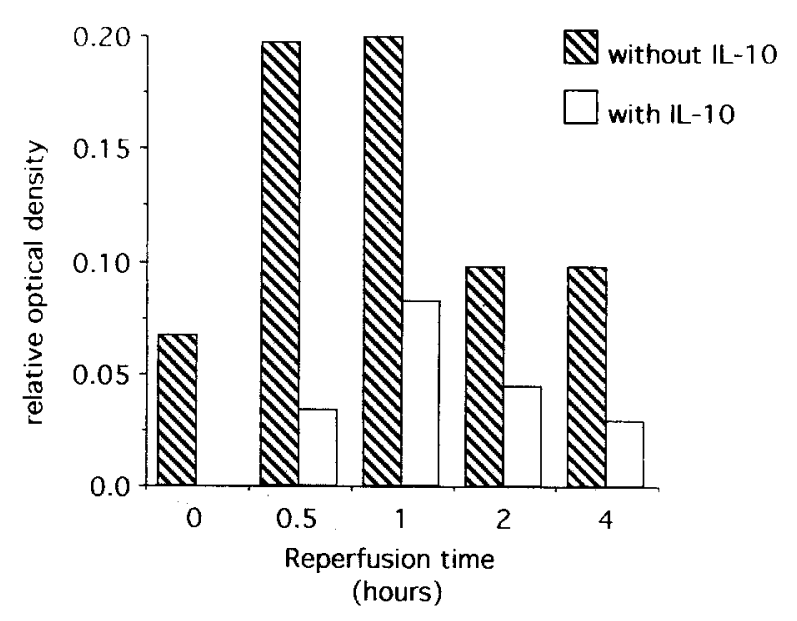

Fig. 4. Densitometry readings from autoradiograph of Northern blot of whole-lung RNA probed with radiolabeled complementary deoxyribonucleic acid specific for rat TNF- $\alpha$. Higher values indicate greater relative amounts of TNF- $\alpha$ messenger RNA. Time 0 represents normal rat lung. All other animals underwent 90 minutes of isolated left lung ischemia and the indicated reperfusion time with or without the addition of $10 \mu \mathrm{g}$ recombinant mouse IL-10 before ischemia.

tion by alveolar macrophages obtained from ischemic lungs and point to at least one mechanism both for the significant lung injury that occurs as a result of ischemia and reperfusion and for the reduction in that injury afforded by IL-10.

TNF- $\alpha$ mRNA production. There was a substantial upregulation of messenger RNA for TNF- $\alpha$ early in the reperfusion time course (Fig. 4). In animals receiving recombinant IL-10 before the induction of ischemia, there was a consistent decrease in the amount of TNF- $\alpha$ messenger RNA detected by Northern blot analysis, indicating that the effects of IL- 10 on TNF- $\alpha$ production occurred at the messenger RNA level, either through inhibition of transcription or through destabilization of the messenger RNA with resultant lower levels of message.

\section{Comment}

We investigated the effects of IL-10 in an in vivo model of single-lung ischemia-reperfusion injury. With this model, we previously found significant injury to the ischemic-reperfused lung occurring in a biphasic pattern during the first 4 hours of reperfusion. ${ }^{6}$ In addition, we determined that the injury that occurs is mediated at least in part by the production of TNF- $\alpha$ during the reperfusion period. ${ }^{7}$ Other 
investigators have shown that IL-10 can downregulate the production of several early-phase inflammatory cytokines, including TNF- $\alpha .{ }^{4,8}$

We found that the administration of recombinant IL-10 before ischemia resulted in a marked decrease in the level of lung ischemia-reperfusion injury, as determined by microvascular permeability detected with radiolabeled BSA. These results correlate with those of others, who have shown that IL-10 is protective in such settings of acute inflammatory upregulation endotoxemia. ${ }^{9}$ Of particular interest in the setting of organ transplantation is the finding that IL-10 can help prevent the development of acute rejection. ${ }^{10,11}$

The ability to administer exogenous agents to prevent reperfusion injury and organ rejection is attractive, but the finding that a substance already endogenously produced has this effect is even more attractive. IL-10 is rapidly upregulated after reperfusion of a transplanted liver ${ }^{5,12}$; this upregulation occurs in a period that suggests most of the stimulus occurs as a result of reperfusion itself, and not as a result of organ rejection. We found in our model that blocking endogenously produced IL-10 with specific antibodies resulted in a higher level of lung reperfusion injury, suggesting that IL-10 may already be functioning in a homeostatic fashion to offset the proinflammatory cytokines and reduce overall injury.

Other investigators have shown good evidence that tissue macrophages may be the source of IL-10 production during the early reperfusion period ${ }^{5}$ and that endogenous $\mathrm{LL}-10$ may function in an autocrine fashion to reduce the level of inflammatory cytokines produced by stimulated macrophages. In this study, we demonstrated that alveolar macrophages stimulated by lung ischemia produced significantly higher levels of the inflammatory cytokine TNF- $\alpha$, and that this production could be attenuated by the addition of IL-10 to the culture medium. This reduction in TNF- $\alpha$ production seems to occur at the messenger RNA level because IL-10 administration before the initiation of ischemia results in consistent decreases in detectable TNF- $\alpha$ messenger RNA in lung tissue during the reperfusion time period.

The finding of an endogenously produced mediator of reperfusion injury and graft rejection is a significant advance in our understanding of the immune mechanisms associated with these phenomena and with the induction of graft tolerance. More sophisticated adjustments of the immune response in the setting of organ transplantation may lead to improved survival with decreased complications.

\section{REFERENCES}

1. Haydock DA, Trulock EP, Kaiser LR, et al. Lung transplartation: analysis of thirty-six consecutive procedures performed over a twelve-month period. J Thorac Cardiovasc Surg 1992;103:329-40.

2. Pham S, Yoshida Y, Aeba R, et al. Interleukin-6, a marker of preservation injury in clinical lung transplantation. J Heart Lung Transplant 1992;11:1017-24.

3. Fiorentino DF, Bond MW, Mosmann TR. Two types of mouse T helper cell. IV. Th2 clones secrete a factor that inhibits cytokine production by Th1 clones. J Exp Med 1989;170:2081-95.

4. Fiorentino DF, Zlotnik A, Mosmann TR, Howard M, O'Garra A. IL-10 inhibits cytokine production by activated macrophages. J Immunol 1991;147:3815-22.

5. Le Moine O, Marchant A, Durand F, Ickx B, Pradier O, Belghiti $\mathbf{J}$, et al. Systemic release of interleukin-10 during orthotopic liver transplantation. Hepatology 1994;20:889-92.

6. Eppinger MJ, Jones ML, Deeb GM, Bolling SF, Ward PA. Pattern of injury and the role of neutrophils in reperfusion injury of rat lung. J Surg Res 1995;58:713-8.

7. Eppinger MJ, Jones ML, Deeb GM, Bolling SF, Ward PA. The role of tumor necrosis factor-alpha in a rat lung model of ischemia-reperfusion injury. Surg Forum 1994;45:297-300.

8. de Waal Malefyt R, Abrams J, Bennett B, Figdor CG, de Vries JE. Interleukin 10 (IL-10) inhibits cytokine synthesis by human monocytes: an autoregulatory role of IL-10 produced by monocytes. J Exp Med 1991;174:1209-20.

9. Howard M, Muchamuel T, Andrade S, Menon S. Interleukin 10 protects mice from lethal endotoxemia. J Exp Med 1993; $177: 1205-8$.

10. Chao S-H, Chu S-H, Lee S-C, Lin J-K, Chen K-M. Interleukin-10 (IL-10) prevents primary nonfuction in allogeneic islet transplantation. Transplant Proc 1995;27:602-4.

11. Lowry RP, Konieczny B, Alexander D, Larsen C, Pearson T, Smith $\mathrm{S}$, et al. Interleukin-10 eliminates anti-CD3 monoclonal antibody-induced mortality and prolongs heart allograft survival in inbred mice. Transplant Proc 1995;27:392-4.

12. Lang T, Krams SM, Villanueva JC, Cox K, So S, Esquivel C, et al. Distinct patterns of Th2 cytokine production during immune activation in pediatric liver allograft recipients. Transplant Proc 1995;27:1146-7.

\section{Discussion}

Dr. Richard J. Novick (London, Ontario, Canada). I congratulate you on excellent work, which we have been following for several years now. I have three questions. First, were the animals heparinized before hilar crossclamping? Second, IL-10 decreases the production of a wide array of cytokines, as recently reviewed in the Journal of Experimental Medicine (1991;174:1209-20). Have you studied other cytokines, aside from TNF- $\alpha$, with respect to their reduced production after IL-10 administration? Third, have you confirmed your work in a larger animal model with respect to the protective effects of IL-10 on ischemia-reperfusion lung injury?

Dr. Eppinger. In response to your first question, the animals were heparinized. Five minutes before the start of 
ischemia, they received $50 \mathrm{U}$ heparin, $150 \mathrm{U} / \mathrm{kg}$, which was allowed to circulate before the hilar structures were crossclamped. Other rat models of inflammation have found similar effects of IL-10 on other cytokines, but such work has not yet been done with this model.

Dr. Thomas M. Egan (Chapel Hill, N.C.). I have one question about your model. Presu.nably this is a recoverable injury, and you chose 4 hours because of the maximal effect. What is the time course of recovery with respect to the microvascular injury? Have you looked at whether your albumin leakage diminishes with time after 4 hours?

Dr. Eppinger. I have not done a lot of work after 4 hours. The only other set of animals that I have examined is at 2 days, when there is almost complete resolution of the leak and the histologic aspect looked essentially normal.

Dr. Egan. What evidence do you have that the TNF- $\alpha$ production that you are seeing is from alveolar macrophages?

Dr. Eppinger. The $\mathrm{TNF}-\alpha$ levels that we measured with the WEHI assay were on extracted alveolar macrophages. They were selectively plated and had harvesting of supernatants. I am sure that there are other cell types making TNF $-\alpha$ but this was the easiest way to look at it.

Dr. Yoshifumi Naka (New York, N.Y.). I have two questions. First, how did you select this dose of IL-10? The second question is related to the first. Did you measure the IL-10 levels in the blood of the control animals?

Dr. Eppinger. The dose of IL-10 was chosen on the basis of some work with mouse models to get a serum level of $250 \mathrm{ng} / \mathrm{ml}$. We did not specifically measure IL-10 levels in the blood of these animals. 